\title{
Parental Separation and Parental Mental Health in Childhood and Risk of Insomnia in Adulthood among Patients with Tinnitus
}

DOI: $10.3766 /$ jaaa.19023

\author{
Hashir Aazh* \\ Basant K. Puri $\dagger$ \\ Brian C. J. Moore
}

\begin{abstract}
Background: Many patients seeking help for tinnitus also suffer from insomnia. Adverse childhood experiences may affect the likelihood of insomnia in later life for such patients.

Purpose: To explore whether parental separation and parental mental health during childhood are related to the severity of insomnia among patients with tinnitus and/or hyperacusis seen in an Audiology clinic.

Research Design: This was a retrospective cross-sectional study.

Study Sample: One hundred seventy-four consecutive patients who attended a tinnitus/hyperacusis clinic in the United Kingdom were included.

Data Collection: Data were based on responses to questionnaires for people seeking help for tinnitus.

Results: Sixteen percent of patients (27/174) reported that during the first 18 years of life, their parents were separated or divorced and $41 \%(72 / 174)$ reported that their parent(s) suffered from a mental illness. The mean score for the insomnia severity index (ISI) was not significantly affected by parental separation or divorce. However, the mean ISI score was significantly worse for patients whose parents had a mental illness. A multinomial logistic regression model, adjusted for the presence of hyperacusis, hearing loss, age, and gender, indicated that for individuals experiencing tinnitus, a history of parental mental illness during their childhood increased the chance of severe insomnia by a factor of 3.4 (95\% confidence interval: 1.1 to $10.8, p=0.04)$. The risk of having severe insomnia was 3.8 times greater for patients with hyperacusis than for those without.
\end{abstract}

Conclusions: Among patients seeking help for tinnitus/hyperacusis, poor parental mental health was associated with severe insomnia.

Key Words: childhood experience, insomnia, mental health, tinnitus

Abbreviations: $\mathrm{BSA}=$ British Society of Audiology; $\mathrm{CBT}=$ cognitive behavioral therapy; $\mathrm{Cl}=$ confidence interval; $\mathrm{HT}$ = hearing threshold; ISI = Insomnia Severity Index; NHS = National Health Service; OR = odds ratio; PTA = pure-tone average; $\mathrm{RRR}=$ relative risk ratio; SD $=$ standard deviation; ULL = uncomfortable loudness level; ULLmin = average ULL over 0.25, 0.5, 1, 2, 4, and $8 \mathrm{kHz}$ for the ear with the lower average ULL

\section{INTRODUCTION}

$\mathrm{T}$ innitus is the sensation of sound without any external sound source. The relationship between the severity of tinnitus and sleep disturbances has been highlighted by several authors (Tyler and Baker, 1983; Meikle et al, 1984; Folmer and Griest, 2000; Schecklmann et al, 2015; Cronlein et al, 2016). Recent studies suggest that more than $70 \%$ of patients seeking help for tinnitus from a specialized audiology

${ }^{*}$ Audiology Department, Royal Surrey County Hospital NHS Foundation Trust, Guildford, UK; †Department of Medicine, Imperial College London, Hammersmith Hospital, London, UK; $\neq$ Department of Experimental Psychology, University of Cambridge, Cambridge, UK

Corresponding author: Hashir Aazh, Audiology Department, Tinnitus \& Hyperacusis Therapy Specialist Clinic, Royal Surrey County Hospital, Guildford, UK; Email: hashir.aazh@nhs.net 
clinic within the UK National Health Service (NHS) also had insomnia (Aazh et al, 2016; Aazh and Moore, 2017a; Aazh et al, 2017; Aazh and Moore, 2019). It has been reported that the emotional and cognitive distress caused by tinnitus has a significant relationship with the severity of sleep disturbances (Cronlein et al, 2016). It also has been reported that among patients seeking help for tinnitus, depressive symptoms are significantly associated with the severity of insomnia (Aazh et al, 2017; Aazh and Moore, 2019).

Research studies in the field of psychiatry suggest that adverse childhood experiences influence the way that individuals respond to stress later in life (Anda et al, 1999; 2007; 2008a,b; 2010). Parental separation and parental poor mental health are important forms of adverse childhood experiences, with prevalence rates of $23 \%$ and $19 \%$, respectively (Anda et al, 2006). Adverse childhood experiences are known to be associated with an increased risk of poor physical and mental health in adulthood (Iniguez and Stankowski, 2016). Also, there is a growing body of evidence suggesting an association between childhood adversities and sleep disorders in adulthood (Bader et al, 2007; Chapman et al, 2011; Ramsawh et al, 2011; Chapman et al, 2013; Kajeepeta et al, 2015). Previous studies showed that poor parental mental health in childhood was associated with an increased risk of hyperacusis and depression in adulthood among help-seeking tinnitus patients (Aazh et al, 2018; 2019). However, no study to date has assessed whether individuals with a history of parental separation and/or poor parental mental health in their childhood are at greater risk of developing insomnia if they experience tinnitus in adulthood.

The aim of this study was to explore whether parental separation and parental mental health during childhood are related to the severity of insomnia among patients seeking help for tinnitus, as seen in an Audiology clinic.

\section{METHODS}

\section{Study Design and Patients}

This was a retrospective cross-sectional study conducted at the Tinnitus and Hyperacusis Therapy Specialist Clinic, Royal Surrey County Hospital, Guildford, United Kingdom. Data were included for all patients who attended the clinic between April and November 2017 and for whom the self-report questionnaire assessing insomnia had been completed $(\mathrm{n}=$ 174). Demographic data for the patients and responses to the self-report questionnaires were imported from records held at the audiology department.

\section{Parental Separation and Parental Mental Health}

To assess parental separation and parental mental health, two questions from the questionnaire for Ad- verse Childhood Experiences (Felitti et al, 1998; Anda et al, 2006) were used. The questions were introduced by the phrase "While you were growing up during the first 18 years of life..." (p. 247; Felitti et al, 1998). The questions were: (a) were your parents separated or divorced? (b) did your parent(s) have depression or mental illness? The responses were "yes" or "no." The test-retest reliability of responses to these questions is in the range good to excellent (Cohen's kappa: 0.46-0.86) (Dube et al, 2004). For convenience, the period of life up to 18 years of age is referred to hereafter as childhood.

\section{Insomnia Severity Index (ISI)}

The ISI was used to assess the severity of sleep difficulties and their effect on the patient's life. The ISI comprises seven items, each of which is rated on a scale from 0 to 4 . The total score ranges from 0 to 28 . Scores from 0 to 7 indicate no clinically significant insomnia, scores from 8 to 14 indicate slight insomnia, scores from 15 to 21 indicate moderate insomnia, and scores from 22 to 28 indicate severe insomnia (Bastien et al, 2001).

\section{Assessment of Hearing Thresholds (HTs)}

Pure-tone audiograms were measured using the procedure recommended by the British Society of Audiology (BSA, 2011a). All audiometric equipment had been calibrated within the past year. The severity of hearing loss was categorized based on the values of the pure-tone average (PTA) at the frequencies $0.25,0.5,1,2$, and $4 \mathrm{kHz}$, as recommended by the BSA (2011a): mild (20-40 dB HL), moderate (41-70 dB HL), severe (71-95 dB HL), and profound (over $95 \mathrm{~dB} \mathrm{HL}$ ). Audiograms were available only for 162 of the 174 patients.

\section{Assessment of Hyperacusis}

Hyperacusis is intolerance of everyday sounds that causes significant distress and impairment in social, occupational, recreational, and other day-to-day activities (Aazh et al, 2016). The sounds may be perceived as uncomfortably loud, unpleasant, frightening, or painful (Tyler et al, 2014). Uncomfortable loudness levels (ULLs) were measured following the BSA recommended procedure (BSA, 2011b). The diagnosis of hyperacusis was based on the average ULL over 0.25 , $0.5,1,2,4$, and $8 \mathrm{kHz}$ for the ear with the lower average ULL, which is called ULLmin. Hyperacusis was considered as present if ULLmin was $\leq 77 \mathrm{~dB}$ HL (Aazh and Moore, 2017b).

\section{Ethical Approval}

This study was approved by the South-West Cornwall and Plymouth Research Ethics Committee 
and the Research and Development department at the Royal Surrey County Hospital.

\section{Data Analysis}

The data were anonymized before statistical analysis. Descriptive statistics (means and standard deviations [SDs]) for the characteristics of the patients and scores for the self-report questionnaires were calculated. The Wilcoxon rank sum test was used to compare the mean of ISI scores for patients grouped based on the presence/absence of parental separation and based on the presence/absence of parental mental illness. The $p$ value required for statistical significance was set at $p<0.05$. The significant difference in ISI scores found for the presence/absence of parental mental illness was explored using multinomial logistic regression to assess the relative risk ratio (RRR) of having different degrees of insomnia based on the presence/absence of parental mental illness. The RRR is defined as the ratio of the probability of an outcome in an exposed group (with parental mental illness in this case) to the probability of an outcome in an unexposed group (without parental mental illness). The values of the $R R R$ and their 95\% confidence intervals (CIs) were calculated both unadjusted and adjusted for the presence of hyperacusis, hearing loss, age, and gender. The STATA program (version 13; StataCorp, College Station, TX) was used for statistical analyses.

\section{RESULTS}

\section{Characteristic of the Study Population}

The average age of the patients was 52 years $(\mathrm{SD}=$ 15.5 years, range 18-84 years). Forty-seven percent (81/174) of the patients were male. The mean PTA across ears was $21 \mathrm{~dB}$ HL $(\mathrm{SD}=13 \mathrm{~dB})$. The mean value of ULLmin was $76 \mathrm{~dB}$ HL (SD = 11). The means and SDs of the HTs and ULLs for each ear and each frequency are shown in Table 1.

Based on the PTA for the better ear, $63 \%$ of patients (102 of 162 with audiograms) had no hearing loss, $33 \%$ (53/162) had mild hearing loss, and 4\% (7/162) had moderate hearing loss. None of the patients had severe or profound hearing loss. The mean ISI score was 13 (SD = 7.7). Based on the values of ULLmin, 33\% of patients (41/124) had ULLs of $77 \mathrm{~dB}$ HL or below, which indicates hyperacusis.

Based on scores for the ISI, $29 \%(51 / 174)$ of the patients did not have insomnia, 28\% (49/174) had mild insomnia, 25\% (44/174) had moderate insomnia, and $17 \%$ (30/174) had severe insomnia.

\section{Parental Separation and Its Relationship with Insomnia}

Sixteen percent of patients (27/174) reported that their parents were separated or divorced during the patient's childhood. The mean ISI score was 13.0 $(\mathrm{SD}=7.8)$ for patients whose parents remained together and 12.6 $(\mathrm{SD}=8)$ for patients whose parents were separated or divorced. The difference was not statistically significant ( $p=0.8$ ).

\section{Parental Mental Health and Its Relationship with Insomnia}

Forty-two percent of patients (72/174) reported that one or both of their parents suffered from a mental illness during the patient's childhood. The mean ISI score was 14.6 $(\mathrm{SD}=7.8)$ for patients whose parents had a mental illness and 11.9 $(\mathrm{SD}=7.5)$ for patients whose parents did not have a mental illness. This difference was statistically significant ( $p=0.029$ ).

Table 1. Means (and Standard Deviations) of HTs and ULLs, Both in dB HL, for Each Ear of the Study Population

\begin{tabular}{|c|c|c|c|c|c|c|c|c|}
\hline \multicolumn{9}{|c|}{ Frequency $(\mathrm{kHz})$} \\
\hline & 0.25 & 0.5 & 1 & 2 & 3 & 4 & 6 & 8 \\
\hline HT right & $\begin{array}{c}19 \\
(13) \\
\mathrm{n}=162\end{array}$ & $\begin{array}{c}18 \\
(13) \\
\mathrm{n}=162\end{array}$ & $\begin{array}{c}17 \\
(14) \\
\mathrm{n}=162\end{array}$ & $\begin{array}{c}19 \\
(16) \\
n=161\end{array}$ & $\begin{array}{c}26 \\
(20) \\
n=123\end{array}$ & $\begin{array}{c}30.5 \\
(21) \\
\mathrm{n}=162\end{array}$ & $\begin{array}{c}37 \\
(24) \\
\mathrm{n}=125\end{array}$ & $\begin{array}{c}38 \\
(27) \\
\mathrm{n}=161\end{array}$ \\
\hline HT left & $\begin{array}{c}19 \\
(14) \\
n=161\end{array}$ & $\begin{array}{c}19 \\
(15) \\
n=161\end{array}$ & $\begin{array}{c}18 \\
(17) \\
\mathrm{n}=161\end{array}$ & $\begin{array}{c}21 \\
(18) \\
n=160\end{array}$ & $\begin{array}{c}28 \\
(23) \\
n=123\end{array}$ & $\begin{array}{c}32 \\
(23) \\
n=60\end{array}$ & $\begin{array}{c}40 \\
(25) \\
n=123\end{array}$ & $\begin{array}{c}40 \\
(27) \\
\mathrm{n}=160\end{array}$ \\
\hline ULL right & $\begin{array}{c}77 \\
(11) \\
\mathrm{n}=124\end{array}$ & $\begin{array}{c}76 \\
(12) \\
\mathrm{n}=136\end{array}$ & $\begin{array}{c}78 \\
(10) \\
\mathrm{n}=136\end{array}$ & $\begin{array}{c}77 \\
(11) \\
\mathrm{n}=136\end{array}$ & $\begin{array}{c}80 \\
(8) \\
n=89\end{array}$ & $\begin{array}{c}78 \\
(11) \\
\mathrm{n}=136\end{array}$ & $\begin{array}{c}80 \\
(9) \\
\mathrm{n}=90\end{array}$ & $\begin{array}{c}75 \\
(14) \\
\mathrm{n}=124\end{array}$ \\
\hline ULL left & $\begin{array}{c}77 \\
(12) \\
\mathrm{n}=124\end{array}$ & $\begin{array}{c}77 \\
(11) \\
\mathrm{n}=135\end{array}$ & $\begin{array}{c}77 \\
(12) \\
\mathrm{n}=136\end{array}$ & $\begin{array}{c}77 \\
(13) \\
n=135\end{array}$ & $\begin{array}{c}81 \\
(8) \\
\mathrm{n}=88\end{array}$ & $\begin{array}{c}78 \\
(12) \\
\mathrm{n}=135\end{array}$ & $\begin{array}{c}80 \\
(10) \\
n=91\end{array}$ & $\begin{array}{c}76 \\
(14) \\
n=123\end{array}$ \\
\hline
\end{tabular}


The presence or absence of parental mental illness was used in multinomial logistic regression analyses to assess its influence on the RRR of having a specific category of insomnia (relative to the category of no insomnia). Columns 3 and 4 of Table 2 show the relationship between insomnia categories as measured via the ISI (dependent variable) and (a) parental mental health, (b) presence of hyperacusis, and (c) hearing loss, treating each measure separately as an independent variable. Different numbers of patients (n) were included in each model as complete data on all measures were not available for all patients. Columns 5 and 6 of Table 2 show the outcomes of a model including all three independent variables, adjusted for age and gender. This model assessed the relationship between parental mental health and insomnia, taking into account the effect of other variables. For patients seeking help for tinnitus, the presence of parental mental illness during the patients' childhood increased the chance of having severe insomnia (the RRR) by a factor of 3.3 unadjusted $(95 \% \mathrm{CI}=1.3-8.4, p=0.013)$. This remained significant when adjusting the RRR for the effect of the presence of hyperacusis, hearing impairment, age, and gender (95\% CI $=1.1-10.8, p=0.04$ ). In addition, the presence of hyperacusis increased the chance of having severe insomnia by a factor of 3.98 unadjusted (95\% CI $=1.3-12.3, p=0.016)$. The effect of the presence of hyperacusis remained significant when the model was adjusted for the effect of parental mental illness, hearing impairment, age, and gender. Finally, the presence of mild hearing loss in the better ear compared with no hearing loss did not significantly increase the risk of having insomnia. There were only seven pa- tients with moderate hearing loss in the better ear, so this category could not be included in the regression model.

\section{DISCUSSION}

7 he results showed that for patients seeking help for tinnitus, the risk of developing severe insomnia in adulthood was 3.4 times greater if their parents had mental illness during the patients' childhood than if they did not, that is, the RRR was 3.4. These RRRs can be compared with the odds ratios (ORs) reported by Chapman et al (2013) in an epidemiological study of the general population $(\mathrm{n}=25,810)$. They assessed the prevalence of "frequent insufficient sleep," which was defined as present if the individual gave a response " $\geq 14$ days" in response to a question asking about the number of days in the past 30 days when they did not get enough sleep. Chapman et al (2013) found that the OR of frequent insufficient sleep was 1.68 (95\% CI $=1.47-1.92)$ when mental illness in the whole family (siblings and parents) was taken into account, which is smaller than the RRR found in our study based on mental illness in the parents alone. Chapman et al (2013) found that the OR of frequent insufficient sleep was $1.23(95 \% \mathrm{CI}=1.08-1.40)$ for individuals whose parents were separated or divorced during childhood relative to individuals whose parents remained together. The significant effect of parental separation found by them contrasts with the lack of significant effect found in this study. This is probably a consequence of the lower statistical power of the present study resulting from the smaller number of participants, which increased the likelihood of a type II error.

Table 2. Outcomes of a Multinomial Logistic Regression Model Assessing the RRR of Having Various Different Severities of Insomnia Based on the Presence/Absence of Parental Mental Illness in Childhood, Hyperacusis (ULLmin $\leq 77 \mathrm{~dB} \mathrm{HL}$ ), and Mild Hearing Loss (PTA 20-40 dB HL) in the Better Ear

\begin{tabular}{|c|c|c|c|c|c|}
\hline Severity of Insomnia & & $\begin{array}{c}\text { Non-Adjusted RRR } \\
(95 \% \mathrm{Cl})\end{array}$ & $p$ Value & $\begin{array}{c}\text { Adjusted RRR } \\
(95 \% \mathrm{Cl})\end{array}$ & $p$ Value \\
\hline No insomnia & Parental mental health illness present & 1 & & 1 & \\
\hline Slight insomnia & & $1.6(0.72-3.7)$ & 0.23 & $1.2(0.42-3.3)$ & 0.75 \\
\hline Moderate & & $1.4(0.59-3.2)$ & 0.45 & $1.4(0.48-3.9)$ & 0.55 \\
\hline Severe insomnia & & $\begin{array}{c}3.3(1.3-8.4) \\
n=174\end{array}$ & 0.013 & $\begin{array}{c}3.4(1.1-10.8) \\
n=124\end{array}$ & 0.04 \\
\hline No insomnia & Hyperacusis present (ULLmin $\leq 77 \mathrm{~dB} \mathrm{HL}$ ) & 1 & & 1 & \\
\hline Slight insomnia & & $1.3(0.4-3.8)$ & 0.67 & $1.3(0.4-4.1)$ & 0.66 \\
\hline Moderate insomnia & & $1.8(0.6-5.2)$ & 0.29 & $1.8(0.6-5.5)$ & 0.32 \\
\hline Severe insomnia & & $\begin{array}{c}3.98(1.3-12.3) \\
n=124\end{array}$ & 0.016 & $\begin{array}{c}3.8(1.1-12.8) \\
n=124\end{array}$ & 0.034 \\
\hline No insomnia & Mild hearing loss (PTA of 20-40 dB HL) & 1 & & 1 & \\
\hline Slight insomnia & & $0.6(0.23-1.3)$ & 0.19 & $0.5(0.16-1.4)$ & 0.17 \\
\hline Moderate insomnia & & $0.5(0.20-1.2)$ & 0.12 & $0.7(0.22-2.1)$ & 0.51 \\
\hline Severe insomnia & & $\begin{array}{c}0.7(0.25-1.8) \\
n=162\end{array}$ & 0.46 & $\begin{array}{c}0.98(0.3-3.4) \\
n=124\end{array}$ & 0.98 \\
\hline
\end{tabular}

Note: The table shows both the unadjusted RRR values and the RRR values adjusted for all variables in the model as well as age and gender, together with $95 \%$ Cls. Significant $p$ values are indicated in bold font. The number of patients included in each analysis is indicated by $\mathrm{n}$. 
In another survey of the general population $(\mathrm{n}=$ 17,337), Chapman et al (2011) assessed sleep disturbances by asking two questions: (a) Have you ever had or do you now have trouble falling asleep or staying asleep? (b) Have you ever had or do you now feel tired even after a good night's sleep? They also asked about various types of childhood adverse events. The ORs for having trouble falling asleep/staying asleep were 1.6 (95\% CI $=1.5-1.8)$ for mental illness in the household and $1.2(95 \% \mathrm{CI}=1.1-1.3)$ for parental separation/divorce. The ORs of feeling tired after a good sleep were $1.6(95 \% \mathrm{CI}=1.5-1.8)$ for mental illness in the family and $1.3(95 \% \mathrm{CI}=1.2-1.4)$ for parental separation/divorce. Thus, the ORs for parental mental illness were greater than the ORs for parental separation, as found in the present study.

The lower ORs for the effects of family mental illness on insomnia found by Chapman et al (2011, 2013) relative to the RRR found here probably reflect the fact that we studied people seeking help for tinnitus, whereas they examined the general population. Tinnitus itself is a risk factor for insomnia (Cronlein et al, 2007), and the combination of tinnitus and a parental history of mental illness may pose a greater risk than parental mental illness alone. However, the differences across studies may also be partly a consequence of differences in the ways that sleep problems were assessed.

Parental mental illness can be considered as part of a larger class of adverse childhood experiences, which are defined as stressful or traumatic life events during the first 18 years of life, including exposures to different form of abuse (physical, emotional, and sexual) and family dysfunction (substance abuse, mental illness, mother treated violently, incarcerated household member, and parental separation) (Felitti et al, 1998). An epidemiological study, based on the same participants as for the study of Chapman et al (2011) ( $\mathrm{n}=17,337)$, reported that the risk of sleep disturbances was increased 2.1-fold for individuals with four or more adverse childhood experiences (Anda et al, 2006). In the study of Chapman et al (2011), the OR for the association between mental illness in the family as a whole and trouble falling asleep $(\mathrm{OR}=1.6)$ was comparable with the ORs for both emotional and physical abuse $(\mathrm{OR}=1.7$, 95\% CI $=1.5-1.9$ ). The ORs were smaller for sexual abuse, witnessing domestic violence, and household substance abuse $(\mathrm{OR}=1.3,95 \% \mathrm{CI}=1.2-1.4)$, and household member imprisoned $(\mathrm{OR}=1.1,95 \% \mathrm{CI}=$ 1.0-1.3). The OR for the association between mental illness in the family as a whole and feeling tired after a good sleep $(\mathrm{OR}=1.6)$ was larger than the ORs for emotional and physical abuse $(\mathrm{OR}=1.4,95 \% \mathrm{CI}=1.2-1.5)$, for witnessing domestic violence and household substance abuse (OR $=1.3,95 \% \mathrm{CI}=1.2-1.4)$, for sexual abuse $(\mathrm{OR}=1.2,95 \% \mathrm{CI}=1.0-1.3)$, and for household member imprisoned $(\mathrm{OR}=1.4,95 \% \mathrm{CI}=1.2-1.6)$.
In the study of Chapman et al (2013), the OR for the association between mental illness in the family as a whole and frequent insufficient sleep was 1.68 (95\% $\mathrm{CI}=1.47-1.92$ ), which is comparable with the ORs for physical abuse $(\mathrm{OR}=1.71,95 \% \mathrm{CI}=1.50-1.97)$, verbal abuse $(\mathrm{OR}=1.59,95 \% \mathrm{CI}=1.42-1.79)$, sexual abuse $(\mathrm{OR}=1.66,95 \% \mathrm{CI}=1.44-1.93)$, substance abusing household member $(\mathrm{OR}=1.47,95 \% \mathrm{CI}=1.03-$ 1.65), witnessing domestic violence $(\mathrm{OR}=1.58,95 \%$ $\mathrm{CI}=1.37-1.82$ ), and incarcerated household member $(\mathrm{OR}=1.54,95 \% \mathrm{CI}=1.21-1.95)$.

Overall, it seems that in the general population, a history of family mental health problems during childhood is a risk factor for sleep problems, and this risk is comparable with or greater than the risk associated with other adverse childhood experiences. Our study shows that a parental history of mental illness is an even greater risk factor for insomnia among those seeking help for tinnitus.

To sum up, poor parental mental health in childhood is associated with a markedly higher risk of developing severe insomnia for patients who seek help for tinnitus in their adulthood. To the authors' knowledge, this finding is new, and it may have implications for clinical practice. In the United Kingdom, the main intervention offered to patients who experience tinnitus is cognitive behavioral therapy, which is typically delivered by specialized audiologists (Aazh et al, 2016). The cognitive behavioral therapy is mainly aimed at reducing the severity of side effects of the tinnitus, including insomnia, rather than reducing the tinnitus itself. Based on this study, it might be beneficial for any therapy to address adverse childhood experiences as well as current circumstances and/or to involve appropriate mental health professionals with expertise in traumatic stress in the rehabilitation team when treating patients with tinnitus, severe insomnia, and a history of adverse childhood experiences. Further research is needed to clarify these issues.

Our study was limited to information that was gathered in day-to-day clinics. We only enquired about the presence or absence of parental separation and parental mental illness. Note that this information takes very little time to obtain, and we think that it would be sensible to include questions of this type while taking a routine case history for patients seeking help for tinnitus or hyperacusis. Future research should systematically examine the relationship between the whole range of possible adverse childhood experiences and insomnia in tinnitus patients.

Our results did not show any significant relationship between hearing loss and insomnia. However, some authors have suggested that poorer hearing is associated with poorer sleep (Test et al, 2011; Lim et al, 2017). The discrepancy could be related to the small variation in hearing loss categories among patients included in 
our study; the majority of our sample had either normal hearing or mild hearing loss. Further work is needed in this regard.

Our results showed that the presence of hyperacusis is significantly associated with severe insomnia. It is possible that this relationship is mediated by a higher prevalence of psychiatric illnesses, mainly depression, among patients with hyperacusis combined with tinnitus compared with tinnitus only (Aazh and Moore, 2017a,c). The link between insomnia and depressive symptoms in patients with tinnitus has been reported by several authors (Cronlein et al, 2016; Xu et al, 2016; Aazh et al, 2017).

\section{CONCLUSIONS}

$\mathrm{P}$ arental separation and parental mental illness among patients seeking help for their tinnitus and/or hyperacusis had a prevalence of $15.5 \%$ and $41.5 \%$, respectively. There were no significant differences in ISI scores between patients with and without a history of parental separation. However, ISI scores were significantly higher (worse) for patients whose parents had a history of mental illness than for those who did not. A regression model showed that the risk of having severe insomnia was 3.4 times greater for patients whose parents had a history of mental illness than for those whose parents did not. Also, the risk of having severe insomnia was 3.8 times greater for patients with hyperacusis than for those without. It may be important to involve mental health professionals with expertise in psychological trauma in the rehabilitation team when treating patients with tinnitus, hyperacusis, severe insomnia, and a history of parental mental illness.

\section{REFERENCES}

Aazh H, Lammaing K, Moore BCJ. (2017) Factors related to tinnitus and hyperacusis handicap in older people. Int J Audiol 56: 677-684.

Aazh H, Danesh A, Moore BCJ. (2019) Parental mental health in childhood as a risk factor for anxiety and depression among people seeking help for tinnitus and hyperacusis. J Am Acad Audiol 30: $772-780$.

Aazh H, Langguth B, Danesh AA. (2018) Parental separation and parental mental health in childhood and tinnitus and hyperacusis disability in adulthood: a retrospective exploratory analysis. Int $J$ Audiol 57:941-946.

Aazh H, Moore BCJ. (2017a) Factors associated with depression in patients with tinnitus and hyperacusis. Am J Audiol 26:562-569.

Aazh H, Moore BCJ. (2017b) Factors related to uncomfortable loudness levels for patients seen in a tinnitus and hyperacusis clinic. Int J Audiol 56:793-800.

Aazh H, Moore BCJ. (2017c) Usefulness of self-report questionnaires for psychological assessment of patients with tinnitus and hyperacusis and patients' views of the questionnaires. Int $J$ Audiol 56:489-498.

Aazh H, Moore BCJ. (2019) Tinnitus loudness and the severity of insomnia: a mediation analysis. Int J Audiol 58:208-212.

Aazh H, Moore BCJ, Lammaing K, Cropley M. (2016) Tinnitus and hyperacusis therapy in a UK National Health Service audiology department: patients' evaluations of the effectiveness of treatments. Int $J$ Audiol 55:514-522.

Anda RF, Brown DW, Dube SR, Bremner JD, Felitti VJ, Giles WH. (2008a) Adverse childhood experiences and chronic obstructive pulmonary disease in adults. Am J Prev Med 34: 396-403.

Anda RF, Brown DW, Felitti VJ, Bremner JD, Dube SR, Giles WH. (2007) Adverse childhood experiences and prescribed psychotropic medications in adults. Am J Prev Med 32:389-394.

Anda RF, Brown DW, Felitti VJ, Dube SR, Giles WH. (2008b) Adverse childhood experiences and prescription drug use in a cohort study of adult HMO patients. BMC Public Health 8:198.

Anda RF, Croft JB, Felitti VJ, Nordenberg D, Giles WH, Williamson DF, Giovino GA. (1999) Adverse childhood experiences and smoking during adolescence and adulthood. JAMA 282: $1652-1658$.

Anda RF, Felitti VJ, Bremner JD, Walker JD, Whitfield C, Perry BD, Dube SR, Giles WH. (2006) The enduring effects of abuse and related adverse experiences in childhood. A convergence of evidence from neurobiology and epidemiology. Eur Arch Psychiatry Clin Neurosci 256:174-186.

Anda R, Tietjen G, Schulman E, Felitti V, Croft J. (2010) Adverse childhood experiences and frequent headaches in adults. Headache 50:1473-1481.

Bader K, Schafer V, Schenkel M, Nissen L, Schwander J. (2007) Adverse childhood experiences associated with sleep in primary insomnia. J Sleep Res 16:285-296.

Bastien CH, Vallieres A, Morin CM. (2001) Validation of the insomnia severity index as an outcome measure for insomnia research. Sleep Med 2:297-307.

BSA. (2011a) Pure-Tone Air-Conduction and Bone-Conduction Threshold Audiometry with and without Masking: Recommended Procedure. Reading, UK: BSA.

BSA. (2011b) Recommended Procedure: Determination of Uncomfortable Loudness Levels. Reading, UK: BSA.

Chapman DP, Liu Y, Presley-Cantrell LR, Edwards VJ, Wheaton AG, Perry GS, Croft JB. (2013) Adverse childhood experiences and frequent insufficient sleep in 5 U.S. States, 2009: a retrospective cohort study. BMC Public Health 13:1-9.

Chapman DP, Wheaton AG, Anda RF, Croft JB, Edwards VJ, Liu Y, Sturgis SL, Perry GS. (2011) Adverse childhood experiences and sleep disturbances in adults. Sleep Med 12:773-779.

Cronlein T, Langguth B, Geisler P, Hajak G. (2007) Tinnitus and insomnia. Prog Brain Res 166:227-233.

Cronlein T, Langguth B, Pregler M, Kreuzer PM, Wetter TC, Schecklmann M. (2016) Insomnia in patients with chronic tinnitus: cognitive and emotional distress as moderator variables. $J$ Psychosom Res 83:65-68.

Dube SR, Williamson DF, Thompson T, Felitti VJ, Anda RF. (2004) Assessing the reliability of retrospective reports of adverse 
childhood experiences among adult HMO members attending a primary care clinic. Child Abuse Negl 28:729-737.

Felitti VJ, Anda RF, Nordenberg D, Williamson DF, Spitz AM, Edwards V, Koss MP, Marks JS. (1998) Relationship of childhood abuse and household dysfunction to many of the leading causes of death in adults. The Adverse Childhood Experiences (ACE) Study. Am J Prev Med 14:245-258.

Folmer RL, Griest SE. (2000) Tinnitus and insomnia. Am J Otol 21:287-293.

Iniguez KC, Stankowski RV. (2016) Adverse childhood experiences and health in adulthood in a rural population-based sample. Clin Med Res 14:126-137.

Kajeepeta S, Gelaye B, Jackson CL, Williams MA. (2015) Adverse childhood experiences are associated with adult sleep disorders: a systematic review. Sleep Med 16:320-330.

Lim HM, Kang W, Park WJ, Jang KH, Ann JS, Moon JD. (2017) Insomnia and hearing impairment among occupational noise exposed male workers. Ann Occup Environ Med 29:36.

Meikle MB, Vernon J, Johnson RM. (1984) The perceived severity of tinnitus. Some observations concerning a large population of tinnitus clinic patients. Otolaryngol Head Neck Surg 92:689-696.
Ramsawh HJ, Ancoli-Israel S, Sullivan SG, Hitchcock CA, Stein MB. (2011) Neuroticism mediates the relationship between childhood adversity and adult sleep quality. Behav Sleep Med 9: 130-143.

Schecklmann M, Pregler M, Kreuzer PM, Poeppl TB, Lehner A, Crönlein T, Wetter TC, Frank E, Landgrebe M, Langguth B. (2015) Psychophysiological associations between chronic tinnitus and sleep: a cross validation of tinnitus and insomnia questionnaires. BioMed Res Int 2015:461090.

Test T, Canfi A, Eyal A, Shoam-Vardi I, Sheiner EK. (2011) The influence of hearing impairment on sleep quality among workers exposed to harmful noise. Sleep 34:25-30.

Tyler RS, Baker JL. (1983) Difficulties experienced by tinnitus sufferers. J Speech Hear Disord 48:150-154.

Tyler RS, Pienkowski M, Rojas Roncancio E, Jun HJ, Brozoski T, Dauman N, Dauman N, Andersson G, Keiner AJ, Cacace AT, Martin N, Moore BC. (2014) A review of hyperacusis and future directions: part I. Definitions and manifestations. Am J Audiol 23:402-419.

Xu Y, Yao J, Zhang Z, Wang W. (2016) Association between sleep quality and psychiatric disorders in patients with subjective tinnitus in China. Eur Arch Otorhinolaryngol 273:3063-3072. 